\title{
Phase Retrieval Quantitative Comparison Between Tilt-series Imaging in TEM and Position-resolved Coherent Diffractive Imaging in STEM
}

\author{
Emanuela Liberti ${ }^{1,2}$, Gerardo Martinez ${ }^{2}$, Colum O’Leary ${ }^{2}$, Peter Nellist ${ }^{2}$ and Angus Kirkland ${ }^{1,2}$ \\ 1. Electron Physical Science Imaging Centre (ePSIC), Diamond Light Source, Harwell Campus \& \\ Innovation Centre, Didcot, Oxfordshire, UK. \\ 2. University of Oxford, Department of Materials, Oxford, UK.
}

Recent advances in fast electron detection and data processing capabilities have triggered a renewed interest in phase retrieval imaging techniques. The common aim of these methods is to recover the phase shift induced by the object, information that would otherwise be lost in the imaging process in which only the intensity of the object wavefunction can be recorded. For weak scattering objects, which introduce only a small phase shift to the electron wave, knowledge of the phase information provides a quantitative measurement of the object potential.

Recent solutions to the phase problem that have benefited from the use of fast detection cameras, include electron ptychography in scanning transmission electron microscopy (STEM) which has successfully been implemented to recover the phase of weak scattering objects [1]. This method uses a fast pixelated detector to record the intensity of a coherent electron diffraction pattern as a function of probe position, resulting in a four-dimensional dataset that is then used to reconstruct the phase via numerical algorithms.

More conventional, well-established, techniques to recover the phase of the object wavefunction record a tilt series [2-3] or a focal series [4] of bright-field TEM images, which can also be used to reconstruct the phase image numerically.

From the principle of reciprocity (for elastic scattering), which states that points in the original detector plane are equivalent to electron sources, recording coherent electron diffraction patterns at every probe position is optically equivalent to recording a tilt series of bright-field images. The principle of reciprocity is schematically shown in Figure 1, where the optical geometry of a tilt-series experiment is shown in comparison to a ptychographic one.

To verify the optical equivalency of a STEM ptychography restoration versus a conventional tilt-series restoration, we have conducted a quantitative comparison of the recovered phase shift of a thin specimen, corresponding to a phase object, such as graphene. Such a specimen favours the application of fast linear algorithms for the numerical restoration of the exit wavefunction, simplifying the imaging formation process. The validity of this equivalency not only aids to our understanding of the physical information encoded in the phase, but also enables a comparison of dose-efficiency for imaging irradiation sensitive materials, such as biological samples, which also can be considered weak phase objects.

In this contribution, we will discuss the experimental phase of graphene restored from a tilt-series of aberration-corrected bright-field images and the equivalent recovered using STEM ptychography at low accelerating voltages, and controlled electron doses. The comparison examines not only the doseefficiency of the two methods, but also presents a quantitative analysis of the phase shift obtained 
following analogous restoration routines [5]. In addition, by analogy to our previous work, which compared quantitatively focal series restoration to STEM ptychography methods [6], we will also show the results of multislice frozen phonon calculations in comparison to the experimental data.

\section{References:}

[1] Yang, H. et al., Nat. Comm. 7 (2016), p. 12532.

[2] Kirkland, A. I. et al., Ultramicroscopy 57 (1995), p. 355.

[3] Haigh, S. J. et al., Phys. Rev. Lett. 103 (2009), p. 16.

[4] Coene, W. M. J. et al., Ultramicroscopy 64 (1996), p. 109.

[5] Rodenburg, J. M. et al., Philosophical Transactions of the Royal Society A: Mathematical, Physical and Engineering Sciences, 339 (1992), p. 521.

[6] Liberti, E. et al., Microscopy and Microanalysis 22 (2016), p. 922.
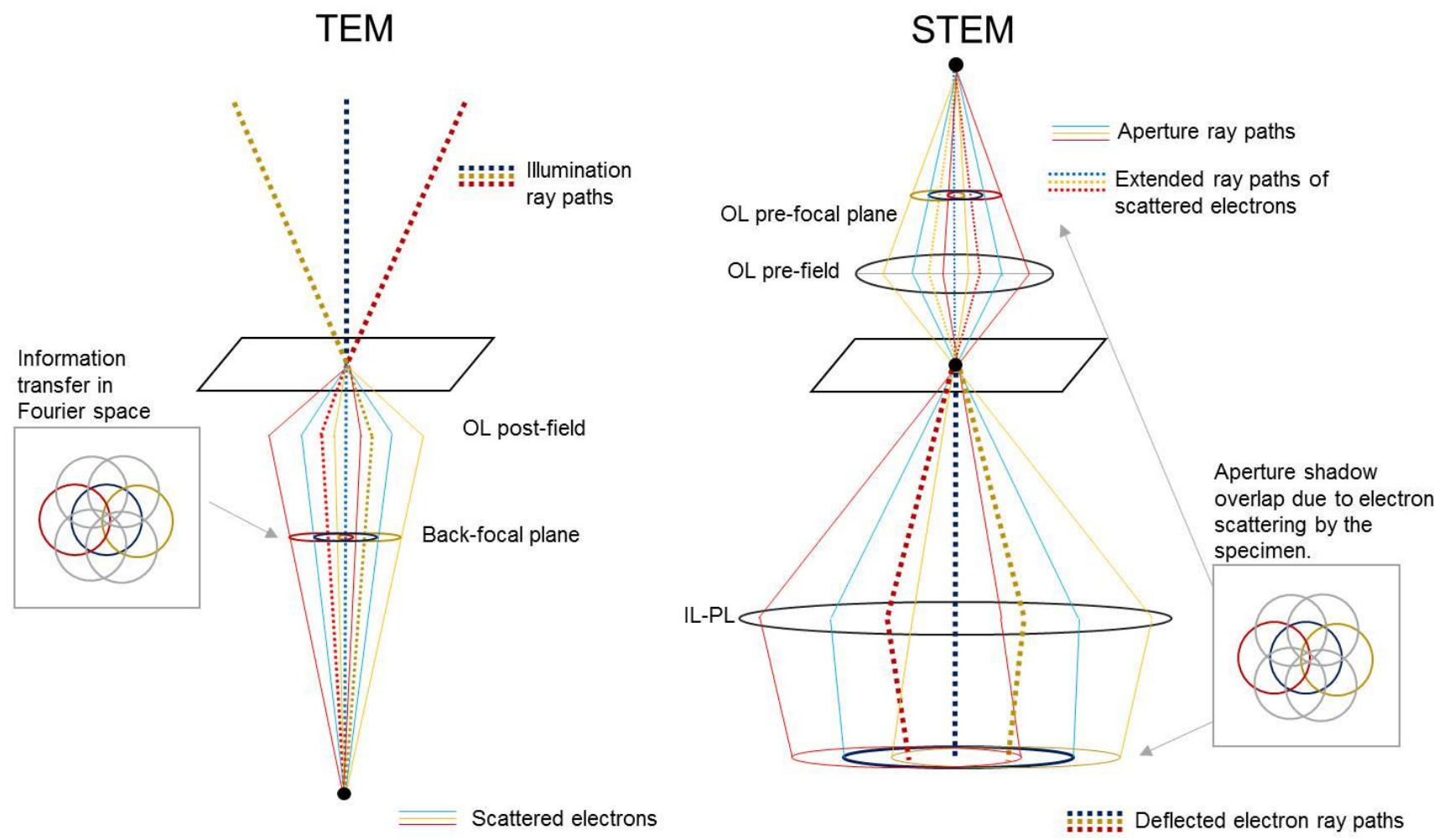

Figure 1. Schematic ray diagram illustrating the principle of reciprocity between tilt-series restoration in TEM, and a ptychographic reconstruction in STEM. In the figure, OL stands for objective lens and IL-PL stands for intermediate projector lens. 